\title{
An electromagnetic, vibration-powered generator for intelligent sensor systems
}

\author{
P. Glynne-Jones, M.J. Tudor*, S.P. Beeby, N.M. White \\ Department of Electronics and Computer Science, University of Southampton, Southampton, SO17 1BJ, Hampshire, UK
}

Received 12 November 2002; received in revised form 26 August 2003; accepted 15 September 2003

\begin{abstract}
This paper describes the design of miniature generators capable of converting ambient vibration energy into electrical energy for use in powering intelligent sensor systems. Such a device acts as the power supply of a microsystem which can be used in inaccessible areas where wires can not be practically attached to provide power or transmit sensor data. Two prototypes of miniature generator are described and experimental results presented. Prototype A is based around two magnets coupled to a coil attached to a cantilever; prototype B is based around four magnets.

For prototype A, experimental results are given for its resonant frequency and its open circuit and loaded output as a function of vibration amplitude. For prototype B, experimental results are given for the generator's Q factor in air and vacuum, its output voltage as a function of vibration amplitude as well as its magnetic field strength. This generator has been tested on a car engine and shown to produce a peak power of $3.9 \mathrm{~mW}$ with an average power of $157 \mu \mathrm{W}$.
\end{abstract}

(C) 2003 Elsevier B.V. All rights reserved.

Keywords: Microgenerator; Energy harvesting; Parasitic power source

\section{Introduction}

There is an increasing level of research activity in the area of alternative power sources for MEMS devices [1] with the terms 'energy harvesting' and 'parasitic power sources' being adopted [2]. A recent review [3] of wireless sensors, covering the area of energy harvesting, indicated the following current areas of research:

- solar,

- vibration,

- temperature difference,

- electromagnetic fields,

- chemical.

Typical application areas for 'self powered' intelligent sensor systems are:

- inside the body (e.g. human, animal),

- on rotating objects,

- within liquids such as molten plastic or setting concrete,

\footnotetext{
* Corresponding author. Tel.: +44-023-8059-4997; fax: +44-023-8059-3709.

E-mail address: mjt@ecs.soton.ac.uk (M.J. Tudor).
}

- structural monitoring such as within bridges, buildings, aircraft or roads,

- environmental monitoring such as pollution monitoring in fields.

This paper describes research at the University of Southampton on an electromagnetic generator targeted at harvesting useful electrical power from ambient vibrations.

Studies performed at the University of Sheffield [4] indicated that power level of $1 \mu \mathrm{W}$ were feasible for a simple spring mass system. Publications from the University of Hong Kong [5,6] have described a single magnet on a laser machined copper spring moving within printed circuit board (PCB) based coil. This paper reports on two and four magnet generators produced at the University of Southampton.

For prototype A, a two magnet generator, experimental results are given for its resonant frequency, the open circuit coil voltage for a range of different amplitudes of base vibration, the load voltage and electrical power across a load resistor on the coil for a fixed base excitation and the load voltage across an optimum load resistance for a range of different amplitudes of base vibration.

For prototype B, a four magnet generator, experimental results are also given for the generator's Q factor in air and vacuum, the output voltage as a function of vibration 
amplitude as well as its magnetic field strength. This generator was also tested in a real application on a car engine.

\section{Microgenerator design and dimensions}

A typical magnet-coil generator consists of a spring-mass combination attached to a magnet or coil in such a manner that when the system vibrates, a coil cuts through the flux formed by a magnetic core. The beam can either be connected to the magnetic core, with the coil fixed relative to the enclosure, or vice versa. Figs. 1 and 2 show the magnet coil geometries investigated.

Design B has been chosen to create a magnetic field through a greater proportion of the length of each coil winding when compared to double or single magnet designs. To improve the degree of coupling, it is important to choose a type of magnet that will produce a strong flux density. Rare earth magnets are ideal for this application, and offer up to five times the magnetic energy density of conventional Alnico magnets. Neodymium Iron Boron $(\mathrm{NdFeB})$ magnets have the most powerful magnetic properties per cubic centimeter $(\mathrm{cm})$ known at this time [7], and can operate at up to $120^{\circ} \mathrm{C}$. If higher temperature operation is required, the less powerful Samarium Cobalt can be used, with a working temperature of up to $250^{\circ} \mathrm{C}$.

The coil is characterised by the proportion of the coil that passes through the magnetic field, the number of turns in the coil, and its series resistance. Second-order effects such as coil inductance can often be ignored due to the low frequency of many applications. A printed coil can be formed by screen

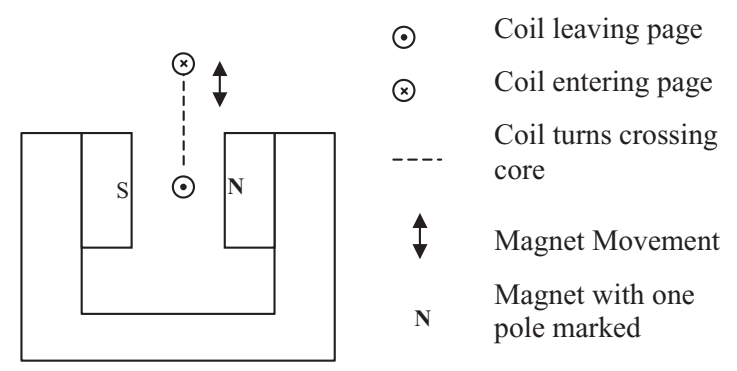

Fig. 1. Electromagnetic generator geometry: prototype A.

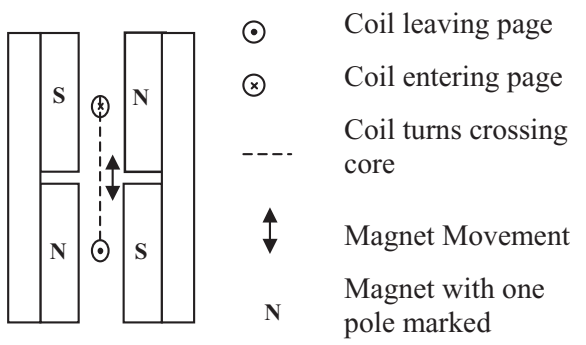

Fig. 2. Electromagnetic generator geometry: prototype B.

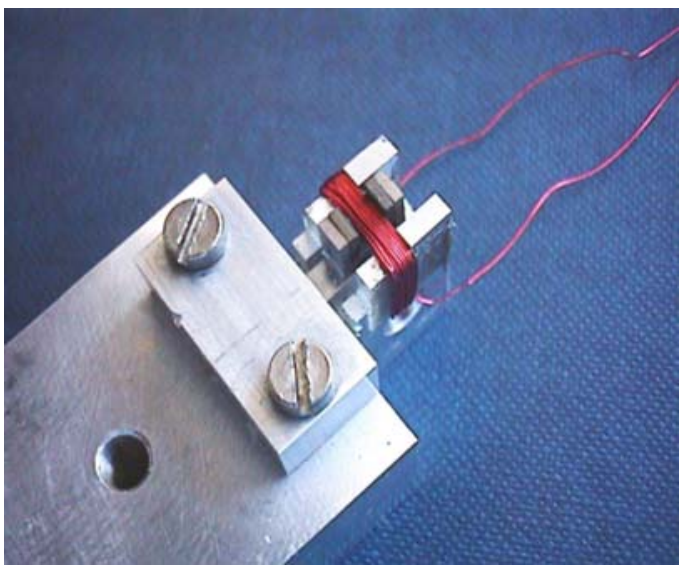

Fig. 3. Photograph of electromagnetic generator geometry: prototype A.

printing layers of conductive materials and insulators onto a substrate using standard thick film technology. A printed coil can be made very thin as printed layers will typically be $10 \mu \mathrm{m}$ thick, making this approach particularly attractive for small-scale devices. The disadvantage of a printed coil is that the small thickness of each layer will result in a high series resistance for the coil. If windings of a larger thickness than are traditionally available from thick-film technology (e.g. $>50 \mu \mathrm{m})$ are required, a wound coil will be more suitable and economic to manufacture.

The configurations shown in Figs. 1 and 2 were modelled to predict power output for various excitations. Figs. 3 and 4 are photographs of prototype devices $\mathrm{A}$ and $\mathrm{B}$. The prototype generators were based around etched stainless steel cantilever to which a hand wound coil was attached to form an inertial mass. NdFeB magnets were held rigidly with respect to the cantilever in an epoxy enclosure.

Prototype device A has a cantilever length of $1.1 \mathrm{~cm}$, a width of $0.9 \mathrm{~cm}$ and a height of $0.85 \mathrm{~cm}$ giving an overall volume of $0.84 \mathrm{~cm}^{3}$. Prototype B has a cantilever length of $2.1 \mathrm{~cm}$, a width of $1.5 \mathrm{~cm}$ and a height of $1 \mathrm{~cm}$ giving and overall volume of $3.15 \mathrm{~cm}^{3}$.

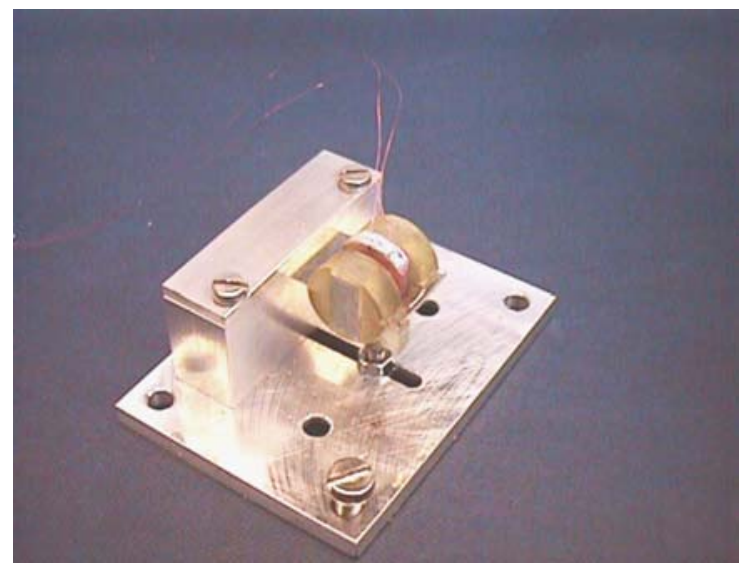

Fig. 4. Photograph of a prototype moving magnet device: prototype B. 


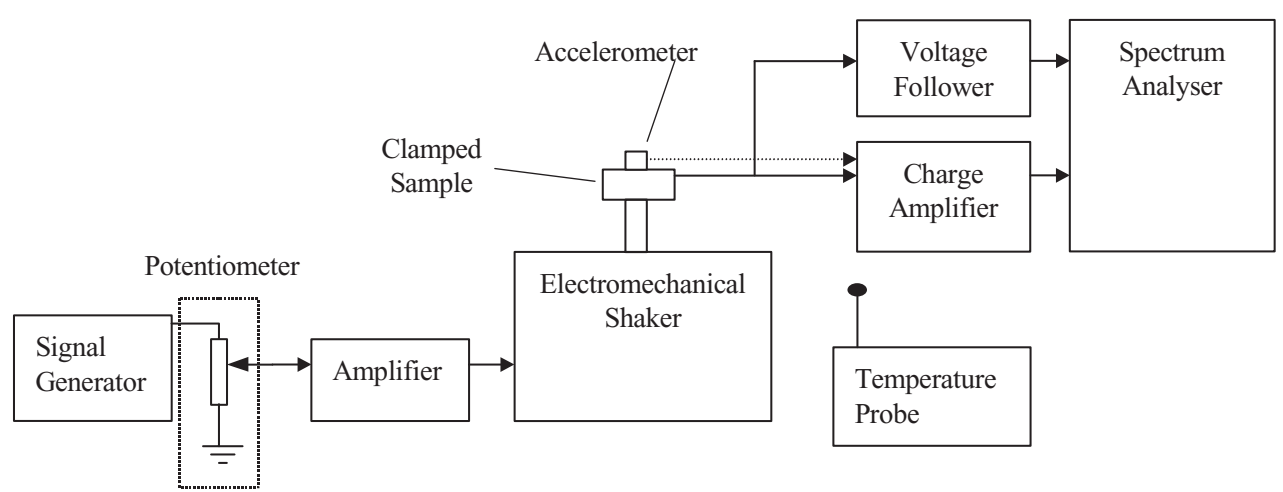

Fig. 5. Experimental setup.

\section{Experimental results}

\subsection{Apparatus and experimental method}

The devices were tested using the shaker and measurement apparatus shown in Fig. 5. A Goodman V.50 Mk.1 (Model 390) Vibration Generator (shaker) was used to supply mechanical vibrations to the samples under test. An accelerometer (Bruel \& Kjaer Accelerometer Type 4369) was mounted to provide data on the amplitude of vibrations applied to the samples, which can not be determined solely from the electrical drive to the shaker, since the shaker has a non-linear response. The accelerometer has a first resonance at $36 \mathrm{kHz}$, which is well above frequencies of interest in the experiments described here. A vernier screw gauge is mounted on a plate that was attached by threaded steel rods to the shaker. The screw gauge has a resolution of $0.01 \mathrm{~mm}$, and allows the tip amplitude of a vibrating beam to be measured. The accuracy of measurements obtained this way is limited by the size of the point at the tip of the gauge, since as the amplitude of the beam changes, the point of contact with the screw gauge will also change.

Many of the experiments described here require that the beam be excited at its resonant frequency. This resonant frequency is sensitive to beam amplitude [8], environmental temperature, and small variations in the clamping position. It is thus hard to achieve resonant excitation with a fixed frequency signal generator and even small frequency errors can introduce significant errors when measuring quantities such as the Q-factor. A closed loop phase-locked-loop (PLL) control circuit was developed to solve this problem.

Before the experiments were performed, calculations were performed based on simple beam theory to determine the maximum beam amplitude that should be allowed to prevent damage through over straining the beam material.

\subsection{Prototype A}

The following experiments were undertaken with prototype A:
1. The resonant frequency was determined and the following experiments were all performed with excitation at the resonant frequency.

2. The open circuit coil voltage was measured for a range of different amplitudes of base vibration.

3. Various load resistors were applied across the coil terminals, and the resulting load voltage, and electrical power measured for a fixed base excitation.

4. The load voltage across an optimum load resistance was measured for a range of different amplitudes of base vibration.

The device was found to have a resonant frequency of $322 \mathrm{~Hz}( \pm 1 \mathrm{~Hz})$. The results of experiments (2)-(4) above are illustrated in Figs. 6, 7 and 8.

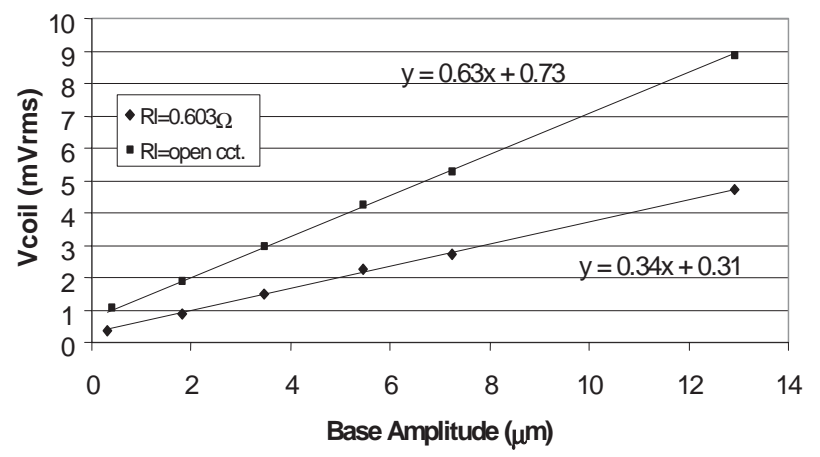

Fig. 6. Coil voltage vs. vibration amplitude, prototype A.

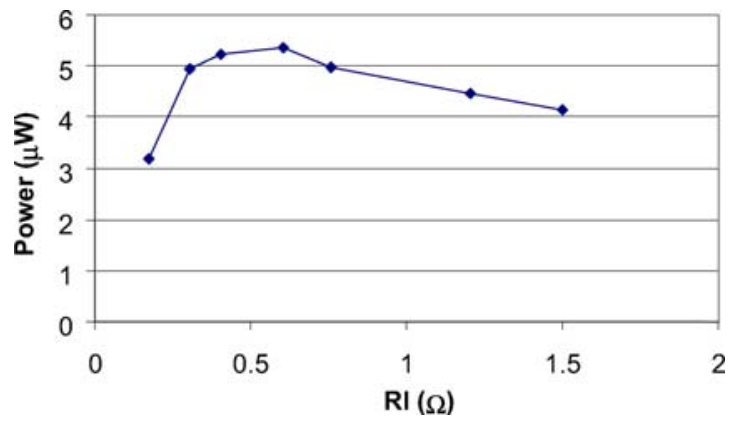

Fig. 7. Power vs. load resistance, base amplitude $=4.4 \mu \mathrm{m}$, prototype A. 


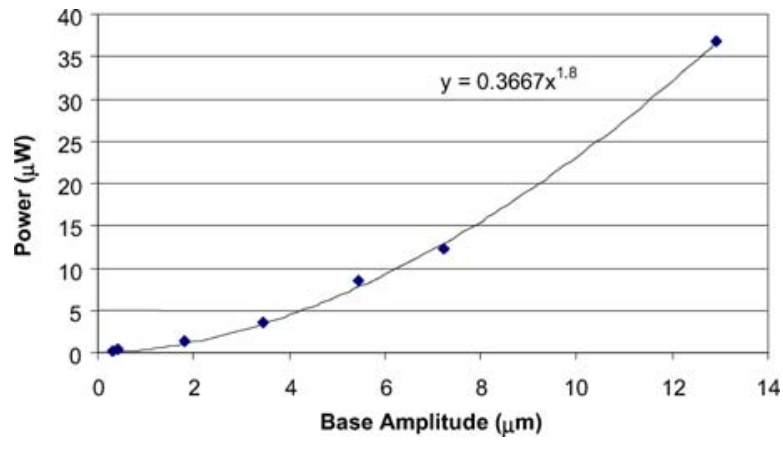

Fig. 8. Power vs. vibration amplitude with optimum load resistance, prototype A.

The graphs show that the coil voltage is a linear function of amplitude, which indicates a uniform and constant magnetic field through the coil. The generator is shown to deliver most power to the load when an optimum load resistance of $0.6 \Omega$ is applied. A maximum power of $37 \mu \mathrm{W}$ was produced at a beam amplitude of $0.36 \mathrm{~mm}$. Beyond this amplitude, gradual shifts in the resonant frequency were observed, indicating that irreversible mechanical changes were occurring in the generator. This maximum amplitude could be increased by improving the mechanical stability of the generator (e.g. improving the bonding between the core and the beam). Tests showed that at a beam amplitude of $0.85 \mathrm{~mm}$, up to $180 \mu \mathrm{W}$ could be produced.

This generator is reasonably small, and produces potentially useful amounts of power when it is shaken sufficiently hard. A major drawback with this design, however, is the very low output voltages developed across the coil. This prototype is also difficult to manufacture in particular the requirement to have the coil pass around the beam. Prototype B seeks to address these problems.

\subsection{Prototype $B$}

To investigate the Q-factor of the prototype $\mathrm{B}$, the logarithmic decrement method was used. In this method the transient response of the beam is examined after it has been set moving by an impulse. If the damping is purely viscous then the amplitude of successive cycles will decay logarithmically. The logarithmic decrement, $\delta$, is defined as

$\delta=\frac{1}{N} \ln \left(\frac{W_{n}}{W_{n+N}}\right)$,

where $N$ is a number of cycles, and $W_{n}$ and $W_{n+N}$ are the amplitudes of cycles separated by $N$ cycles. If the damping factor, $\zeta$, is small then it can be shown [9] that

$\zeta \approx \frac{\delta}{2 \pi}$

and,

$Q=\frac{1}{2 \zeta}$

The circuit shown in Fig. 9 was used.

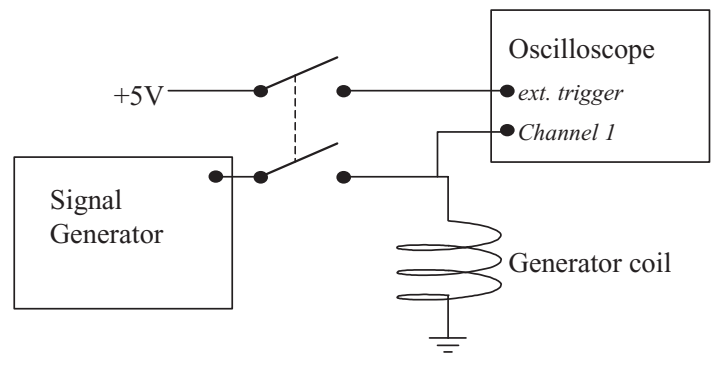

Fig. 9. Q-factor test circuit.

Generators were excited at their resonant frequency using a sinusoidal waveform from the signal generator applied across the coil. An oscilloscope was connected across the electrodes of the coil to monitor the voltage. A double-throw switch is used to simultaneously disconnect the signal generator, and generate a trigger signal to the oscilloscope. When the switch is thrown, the scope triggers, and the decaying waveform can be examined. In each case the decay was measured over a total of 12 cycles, with an initial coil voltage amplitude of $250 \mathrm{mV}$. The design includes a vacuum-sealed cover so that the air surrounding the beam could be evacuated with a vacuum pump.

Table 1 shows the results for three different sized generators each with a different resonant frequency. Each entry represents the average of four readings and an error of around $10 \%$ is associated with each entry. The first two rows show that applying a vacuum to the beams has little measurable effect on the Q-factor. Mechanical support and electrical load damping account for the majority of the damping observed. Material damping is also present but is less significant.

To measure the strength of the magnetic field in the gap of each generator, experiments were performed to measure the open circuit coil voltage as a function of beam amplitude. Fig. 10 shows the results for generators 1 and 2 .

The results show a good degree of linearity, which means that the magnetic field around the coil remains constant over the full range of even the larger amplitudes of vibration shown here. The magnetic field, $B$, is calculated using the formula

$B=\frac{V_{\mathrm{COIL}}}{N l|\dot{x}|}=\frac{V_{\mathrm{COIL}}}{N l A \omega}$,

where $l$ is the length of coil in the field, and $A$ is the amplitude of the displacement, $x$.

Table 2 lists the measured values, and the values predicted using finite element analysis (FEA). The FEA is within $12 \%$ of the measured value.

Table 1

Prototype B Q-factors

\begin{tabular}{llll}
\hline Generator & $\begin{array}{l}\text { Frequency } \\
(\mathrm{Hz})\end{array}$ & $\begin{array}{l}\text { Q-factor } \\
\text { in air }\end{array}$ & $\begin{array}{l}\text { Q-factor in vacuum. } \\
\text { Pressure }=27 \mathrm{~Pa}\end{array}$ \\
\hline 1 & 208 & 121.8 & 111.8 \\
2 & 106 & 141.3 & 145.9 \\
3 & 99 & 86.2 & - \\
\hline
\end{tabular}




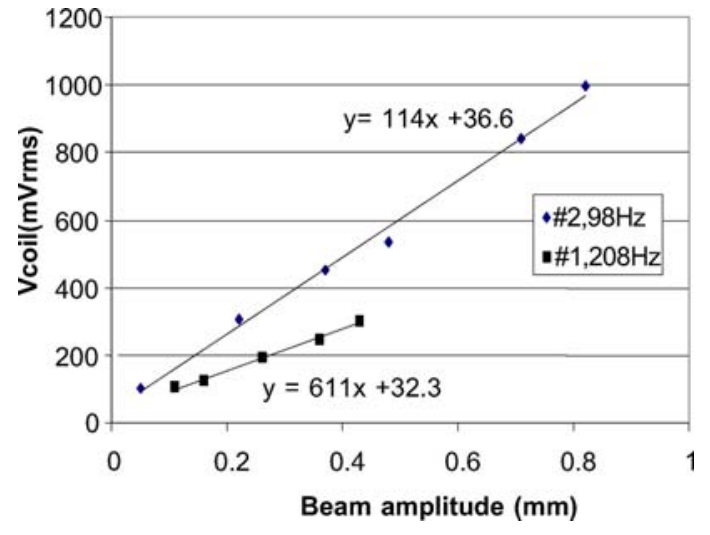

Fig. 10. Coil voltage vs. beam amplitude: prototype B.

Table 2

Magnetic field values

\begin{tabular}{lll}
\hline Generator & \multicolumn{2}{l}{ Magnetic field, $B(\mathrm{~T})$} \\
\cline { 2 - 3 } & Measured & Predicted \\
\hline 1 & 0.192 & 0.214 \\
2 & 0.239 & 0.267 \\
\hline
\end{tabular}

\section{Practical applications}

The following experiment was performed to demonstrate that the technology has the potential to be useful in a practical application. The generator was mounted on the top of the engine block of an, otherwise unmodified, 5-year-old Volkswagen Polo. Experiments showed that the power produced by the generator was largely determined by the engine speed with a resonant peak at around $3000 \mathrm{rev} / \mathrm{s}$, perhaps relating to a resonance in the engine mounting. Fig. 11 shows data taken from a typical short drive in Southampton.

During the three minute journey, $1.24 \mathrm{~km}$ was covered at an average speed of $25 \mathrm{~km} / \mathrm{h}$. The journey included stops at three traffic lights. Over the period, an average power of $157 \mu \mathrm{W}$ was produced, with a peak value of $3.9 \mathrm{~mW}$. This demonstrates that an application in this environment could feasibly generate enough power to perform useful tasks and

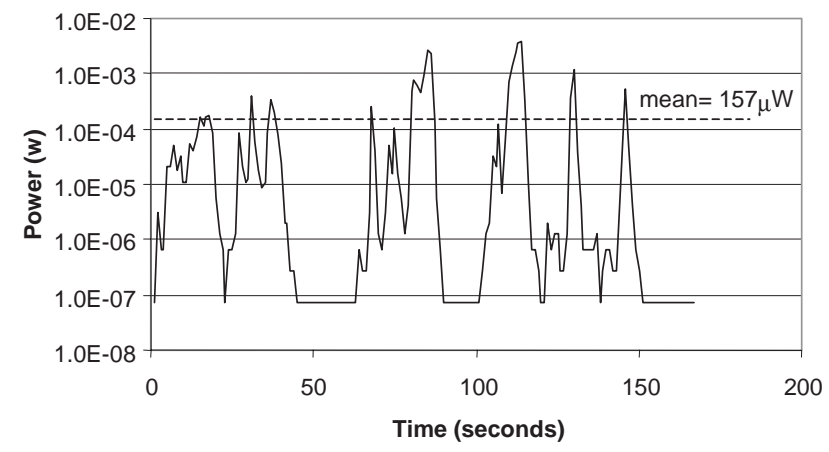

Fig. 11. Output power from electromagnetic generator protoype B on a car engine. further application of this generator is described elsewhere [10].

\section{Conclusions}

An electromagnetic generator based around a moving coil between two magnets is capable of generating useful level of power, however the output voltage is considered too low for practical application and the geometry requires coil winding around the magnets which is cumbersome. A second electromagnetic generator based around a coil between four moving magnets is capable of generating useful power and voltage levels from ambient vibrations. A device has been described which can produce an average power of $157 \mu \mathrm{W}$ when mounted on the engine block of a car.

Further work will examine the translation of this design into a structure compatible with batch fabrication processes as well as investigating concepts for further miniaturisation without loss of conversion efficiency.

\section{Acknowledgements}

The authors wish to thank the Engineering and Physical Sciences Research Council (EPSRC) for their financial support under grant number GR/M35086. We also gratefully acknowledge the support and assistance given to us by Morgan Electroceramics Ltd.

\section{References}

[1] P. Glynne-Jones, N.M. White, Self-powered systems, a review of energy sources, Sens. Rev. 21 (2) (2000) 91-97.

[2] F. Discenzo, Parasitically Powered Sensing Device, US Pattent 6,304,176 (Oct. 16, 2001)

[3] Wireless sensors-buyer beware, April 2001. Available from http://www.sensorsmag.com/articles/0401/18/index.htm.

[4] C.B. Yates, R.B. Williams, Analysis of a micro-electric generator for microsystems, Physica A 52 (1/3) (1996) 8-11.

[5] W.J. Li, Z. Wen, P.K. Wong, G.M.H. Chan, P.H.W. Leong, A micromachined vibration-induced power generator for low power sensors of robotic systems, in: World Automation Congress: 8th International Symposium on Robotics with Applications, June 16-21, 2000, Hawaii, USA.

[6] N. Ching, H. Wong, W. Li, P. Leong, Z. Sar, U. Chongquing, in: A Laser Micromachined Vibrational to Electrical Power Transducer for Wireless Sensing Systems (Transducers 01), June 10-14, 2001, Munich, pp. 42-45.

[7] Eclipse Rare Earth Magnetic Limited. Available from http://www.eclipse-magnetics.co.uk/rare_earth.html.

[8] M.V. Andres, K.W.H. Foulds, M.J. Tudor, Non linear vibrations and hysteresis of micromechanical silicon resonators as frequency out sensors, Electron. Lett. 23 (18) (1987) 952-954.

[9] W.T. Thomson, Theory of Vibration with Applications, third ed., Allen and Unwin, London, 1988.

[10] E.P. James, M.J. Tudor, S.P. Beeby, P. Glynne-Jones, J.N. Ross, N.M. White, A Wireless Self-Powered Micro-System for Condition Monitoring (Eurosensors XVI), Prague, 2002. 


\section{Biographies}

P. Glynne-Jones graduated with first class honours from the School of Electronics and Computer Science at the University of Southampton. He was sponsored through his degree by the Defence Education and Science Group and was an IEE scholar. Peter was awarded a Ph.D. in vibration powered microsystems from the same University in 2001. He is currently in a Zen Buddhist Monastery in Luton.

M.J. Tudor obtained a Ph.D. in Physics from Surrey University and a B.Sc. (Eng.) in Electronic and Electrical Engineering from University College London. In 1987, John joined Schlumberger Industries working first at their Transducer Division in Farnborough and then their Research Centre in Paris, France. In 1990, he joined Southampton University as a Lecturer. In 1994, John moved to ERA Technology becoming the Microsystems Programme Manager. In 2001, John returned to Southampton University as a Senior Research Fellow in the School of Electronics and Computer Science to pursue university based research in microsystems. John has 25 publications and 7 patents and served on the IEE Microengineering Committee for 4 years. He is both a Chartered Physicist and Engineer.

S.P. Beeby (EPSRC Advanced Fellow) has been a Research Fellow/Assistant at Southampton University for 9 years. His Advanced
Fellowship is on the subject of piezoelectric thick-film materials for microelectromechanical systems (MEMS). His Ph.D., 'Mechanical Isolation of Miniature Resonant Sensors and Stress Relieving Packages', was on the subject of micromachined silicon resonators. His research interests include vibration powered microgenerators, microsystems, instrumentation and thick-film materials development. He has 75 publications in the area and is a Chartered Engineer and a Chartered Physicist.

N.M. White holds a personal chair in the School of Electronics and Computer Science, University of Southampton. He has been active in sensor development since 1985. In 1988, he was awarded a Ph.D. from the University of Southampton. He has considerable experience in the design and fabrication of a wide variety of sensors, formulation of novel thick-film sensing materials and intelligent sensor systems. In 1994, he co-authored a book entitled 'Intelligent sensor systems', he was the editor of the proceedings of 'Eurosensors XII', held at Southampton in September 1998 and the conference Chairman of 'Sensors and their Applications X', Cardiff, 1999. Professor White was the Chairman of the Instrument Science and Technology (ISAT) group of the Institute of Physics from 1997 to 1999 . He has over 100 publications in the area of instrumentation and advanced sensor technology. His professional qualifications include Chartered Engineer, Fellow of the IEE, Fellow of the 1OP, Chartered Physicist and Senior Member of the IEEE. 